
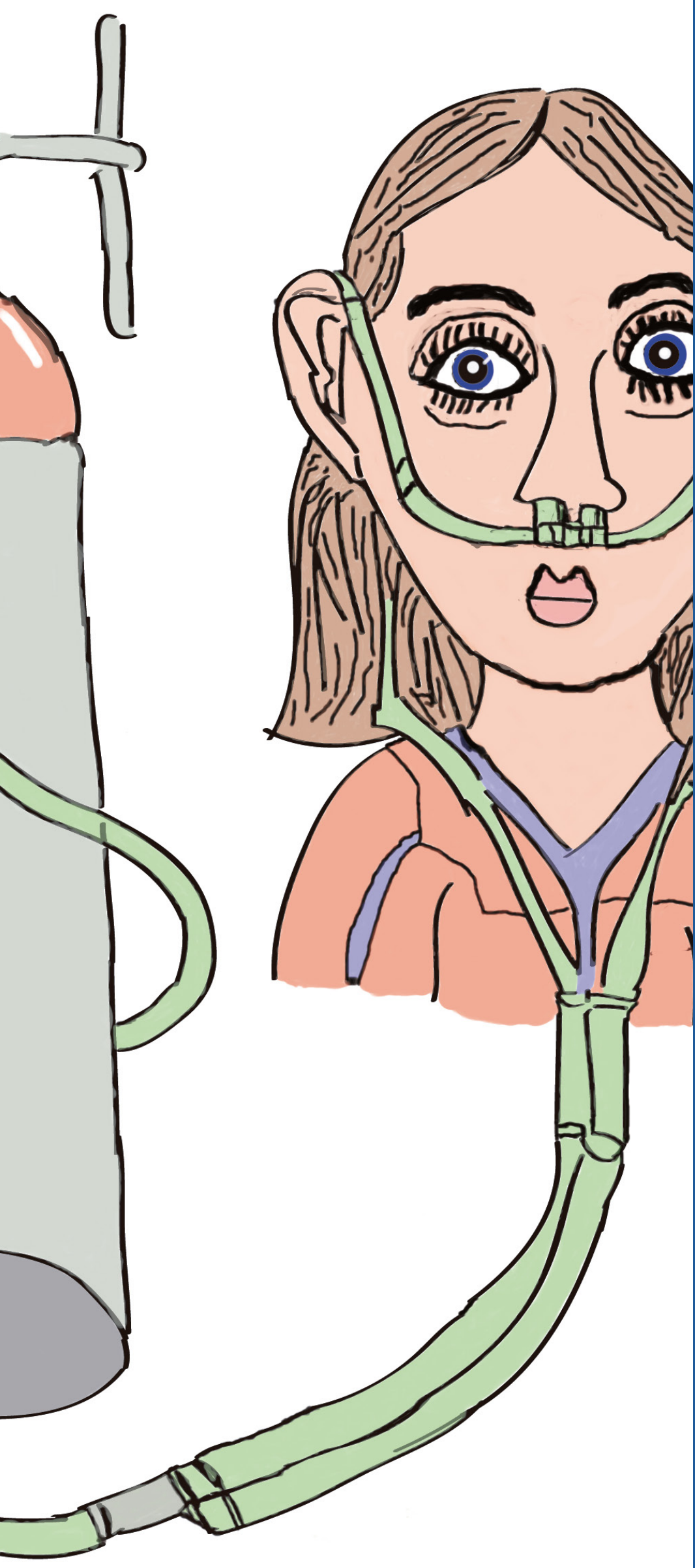

INVESTIGACIÓN CIENTÍFICA Y TECNOLÓGICA

Cómo citar (APA): Betancourt-Peña, J., Torres-Del Castillo, N. \& Hurtado-Gutiérrez, H. (2018). Enfermedad pulmonar intersticial difusa: evaluación clínica y funcional previa a un programa de rehabilitación pulmonar. Revista Colombiana de Rehabilitación, 17 (2), 67-81. https://doi.org/ 10.30788/RevColReh.v17. n2.2018.336

Esta obra se encuentra bajo licencia internacional CC BY 4.0. 


\title{
Enfermedad pulmonar intersticial difusa: evaluación clínica y funcional previa a un programa de rehabilitación pulmonar
}

\author{
Jhonatan Betancourt-Peña ${ }^{\mathrm{abc}}$ \\ ORCID: 0000-0002-7292-7628
}

\author{
Nathalie Torres-Del Castillo \\ ORCID: 0000-0003-2983-4722
}

Hugo Hurtado-Gutiérrez ${ }^{\mathrm{ab}}$

ORCID: 0000-0003-4119-1088

a. Universidad del Valle. Facultad de Salud. Escuela de Rehabilitación Humana.

b. Institución Universitaria Escuela Nacional del Deporte. Facultad de Salud y Rehabilitación.

c. Cardioprevent SAS.

\section{Resumen}

La presencia de enfermedades intersticiales se ha vuelto más frecuente en nuestro medio. La rehabilitación pulmonar es una opción beneficiosa, sin embargo, el comportamiento y estrategias de intervención siguen escasas, justificando su investigación en nuestro país. El objetivo es describir estos pacientes al ingreso de un programa de rehabilitación pulmonar desde la parte sociodemográfica y calidad de vida relacionada con la salud, hasta su parte clínica y funcional. Método: Estudio descriptivo en 41 pacientes que ingresaron a rehabilitación pulmonar, se realizó recolección de datos sociodemográficos, clínicos y farmacológicos; se implementó el test de la marcha de los 6 minutos para valorar la capacidad funcional; la calidad de vida relacionada con la salud fue valorada con St. George's Respiratory Questionnaire y ansiedad y depresión con Hospital Anxiety and Depression Scale. Resultados: Hombres 56,1\%. Edad media 57.6 \pm 12.9 . El hábito de fumar estuvo presente en $39 \%$ de los casos con media de $18.97 \pm 17.2$ paquetes/año. El $66.7 \%$ de los pacientes que visitaron urgencias por complicaciones respiratorias fueron hospitalizados con una media de $9.7 \pm 14.4$ días. La distancia recorrida fue de 348.2 \pm 109 metros. Los hombres recorren en promedio 108.8 metros más que las mujeres. Discusión: Se discuten los resultados a la luz del contexto local y regional teniendo en cuenta la disponibilidad y uso de pruebas diagnósticas. Los resultados contrastan con la literatura respecto al sexo de los pacientes en los que predomina con EPID. Los hallazgos son coherentes con el curso de las enfermedades en cuanto a visita a urgencias y hospitalizaciones.

Palabras clave: Enfermedades pulmonares intersticiales; enfermedades pulmonares; hospitalización; calidad de vida.

\section{Interstitial Lung Disease: clinical and functional evaluation prior to a pulmonary rehabilitation program}

\begin{abstract}
The presence of interstitial diseases has become more frequent in our environment. Pulmonary rehabilitation is a beneficial option. However, the behavior and intervention strategies are still scarce. This justifies the research on these kind of diseases in our country. The objective is to describe the patients' condition upon admission in a pulmonary rehabilitation program considering the socio-demographic and quality of life characteristics related to health as well as the clinical and functional characteristics. Method: Descriptive study made with 41 patients admitted to a pulmonary rehabilitation program. Socio-demographic, clinical and pharmacological data was collected. The 6-Minute Walk Test was used to evaluate the functional capacity. The quality of life related to health was evaluated with St. George's Respiratory Questionnaire and the anxiety, and its relation with depression was evaluated with the Hospital Anxiety and Depression Scale. Results: Men $56.1 \%$. Mean age $57.6 \pm 12.9$. The habit of smoking was present in $39 \%$ of the cases with a mean of $18.97 \pm 17.2$ packs / year. Hospitalization was made on $66.7 \%$ of patients who visited the emergency department due to respiratory complications, with a mean of $9.7 \pm 14.4$ days. The distance traveled was $348.2 \pm 109$ meters. Men walked an average of 108.8 meters more than women. Discussion: The results are discussed within the local and regional context, considering the availability and use of diagnostic tests. The results contrast with the literature regarding the sex of the patients where ILDs predominate. The findings are consistent with the diseases' course in terms of emergency room visits and hospitalizations.
\end{abstract}

Keywords: Interstitial lung diseases; lung diseases; hospitalization; quality of life. 


\section{Introducción}

$\mathrm{L}$

a Enfermedad Pulmonar Intersticial Difusa (EPID) es una variedad de enfermedades clasificadas en tres principales grupos por sus manifestaciones clínicas, alteraciones anatómicas y fisiológicas, patrones radiológicos y compromiso de la función pulmonar con cuadros similares (Raghu et al., 2011).

Es una enfermedad cada vez más visible, no obstante, los datos epidemiológicos disponibles son limitados e imprecisos pues varían en cada región, desconociendo su incidencia, prevalencia y estimación del problema. En España la incidencia en el 2004 fue de 7,6 casos/100.00o habitantes, siendo la Fibrosis Pulmonar Idiopática (FPI) la más frecuente con una cifra de 1,6/100.00o habitantes (López-Campos \& Rodríguez-Becerra, 2004). En el año 2009, la incidencia en Grecia fue de 4,6/100.000 y la prevalencia de 17.3/100,00o habitantes/año (Karakatsani et al., 2009). En Colombia suelen ser frecuentes los casos, considerando la influencia de algunas condiciones laborales, medioambientales y otras presencias patológicas (Torres-Duque, Dueñas \& Caballero, 2007). En 1991 en el hospital Santa Clara de Bogotá, las EPID tuvieron un $17.8 \%$ de las causas de hospitalización con tendencia a incrementar los próximos años (Dueñas, Londoño, Manzano, \& Ojeda, 1991); sin embargo, aún no se dispone de datos sobre incidencia y prevalencia de EPID ni FPI en Colombia (González-García, Chamorro, Jaramillo, Casas, \& Maldonado, 2014).

Las limitadas opciones de tratamiento son destinadas a mejorar la sintomatología de los pacientes; se ha descrito que el tratamiento farmacológico ayuda al control de la sintomatología y el progreso de la enfermedad, es común el uso de anti-fibroticos y glucocorticoides (Fernández, Sánchez, Moreno, \& Fabrellas, 2009). El tratamiento no farmacológico se centra en evitar factores de riesgo como hábito de fumar, químicos y/o factores ambientales. El trasplante pulmonar resulta ser la última opción para estos pacientes siendo una intervención costosa y de poca accesibilidad (Selman \& Undurraga, 2015).

La Rehabilitación Pulmonar (RP) es otra alternativa de tratamiento con nivel de evidencia y recomendación 1B para las enfermedades intersticiales (Selman \& Undurraga, 2015), ofreciendo mejoría en la calidad de vida, sintomatología y capacidad funcional, teniendo en cuenta que los pacientes que ingresan a RP presentan una sintomatología marcada y un avanzado deterioro de su patología (Betancourt-Peña \& Hurtado-Gutiérrez, 2015; Holland et al, 2012); sin embargo, los estudios están enfocados en determinar el resultado final de la RP y pocos han caracterizado la población en cuanto a aspectos sociodemográficos, clínicos, funcionales y de calidad de vida. Sumado a esto, en Colombia y América Latina el acceso a estos programas se complica por el difícil diagnóstico de la enfermedad debido al alto costo y la demanda de personal altamente especializado (Selman \& Undurraga, 2015; Duran-Palomino, Wilches-Luna \& Martínez-Santa, 2010). Esto además, retrasa las posibilidades de avanzar en el conocimiento de estrategias terapéuticas, comportamiento de esta patología y evidencia de los beneficios de la rehabilitación, los cuales dependen de la evolución de la enfermedad y el inicio de la intervención (Holland et al, 2012).

Una valoración y descripción detallada permitirá a profesionales de la salud involucrados en programas de RP implementar intervenciones basadas en las necesidades de los pacientes permitiendo una mejor comprensión de esta patología y su tratamiento. Este estudio tiene como objetivo describir estos pacientes al ingreso de un programa de RP desde los aspectos clínico-funcionales, sociodemográficos y de calidad de vida. 


\section{Método}

Estudio descriptivo de corte transversal

\section{Participantes}

En un periodo de 30 meses ingresaron 44 pacientes al programa de RP, 3 no cumplían con los criterios de inclusión debido a que no tenían Tomografía Axial Computarizada (TAC) al momento de ingresar a la RP. Se obtuvo una muestra por conveniencia de 41 pacientes que ingresaron a un programa de rehabilitación pulmonar durante el periodo bajo estudio. Como criterios de inclusión los pacientes tuvieron diagnóstico médico de EPID comprobado mediante radiografía de tórax y tomografía axial computarizada de alta resolución, y aceptaron la partícipación en el estudio mediante firma de consentimiento informado. Las pruebas de función pulmonar e historia clínica completa sobre factores de riesgo, aspectos clínicos y tratamiento se realizaron como parte de la valoración inicial y como criterio de ingreso de la RP (Raghu et al., 2011; Travis et al., 2013; King Jr, 2005). En los criterios de exclusión se consideraron pacientes con enfermedades crónicas no trasmisibles no controladas, pacientes con limitaciones o alteraciones osteomusculares que alteraran o impidieran realizar el test de caminata de 6 minutos (TC6M), alteraciones cognitivas o afectivas (ansiedad o depresión) que limitaran realizar los cuestionarios de calidad de vida relacionada con la salud (CVRS).

\section{Instrumentos}

Las variables que se tuvieron en cuenta para este estudio fueron: sociodemográficas y clínicas, ansiedad y depresión, calidad de vida relacionada con la salud y capacidad funcional.

Se elaboró un cuestionario de variables sociodemográficas y clínicas para el estudio que incluyó ítems como: sexo, edad, lugar de residencia, estrato socioeconómico y estado civil. Características clínicas: hábito de fumar (número paquetes año) y exposición al humo de leña (número de años de exposición), tipo de EPID, remisión médica, visitas a urgencias, hospitalizaciones en el último año por complicaciones respiratorias, pruebas de función pulmonar con los valores en porcentaje de predicho de la CVF, VEF1, VEF1/CVF, obtenidos en la espirometría al menos 3 meses antes de iniciar RP, índice de masa corporal (IMC), tratamiento farmacológico y uso de oxigeno domiciliario (Betancourt-Peña, Muñoz-Erazo, \& Mora-Guerra, 2015).

La Escala de Medical Research Council (MRC) se utilizó para valorar la severidad de la disnea al esfuerzo o en actividades de la vida diaria (Fletcher, 1960), esta escala presenta una puntuación de o a 4 y el participante selecciona el grado que más representa su situación actual: $\mathrm{o}=$ disnea sólo ante actividad física muy intensa, 1 = disnea al andar muy rápido o al subir un cuesta poco pronunciada, 2 = incapacidad de andar al mismo paso que otras personas de la misma edad, 3 = disnea que obliga a parar antes de los $100 \mathrm{~m}$, a pesar de caminar a su paso y en terreno llano y $4=$ disnea al realizar mínimos esfuerzos en las actividades diarias como vestirse o que impida al paciente salir de su domicilio. Esta escala se ha utilizado frecuentemente en pacientes con EPID para describir los cambios la disnea en las actividades luego de la rehabilitación pulmonar (Jastrzebski, Gumola, Gawlik, \& Kozielski, 2006).

Posteriormente la CVRS se evaluó por medio del cuestionario St. George Respiratory Questionnaire (SGRQ), el cual está compuesto por 50 preguntas agrupadas en 3 dominios: síntomas, actividad e impacto. 
La puntuación total y de cada dominio va de o a 100, donde los valores que se aceran a 100 representan una peor CVRS y los valores que se acercan a o denotan una mejor CVRS, su validación al español sugiere una viabilidad razonable para ser aplicada en pacientes con enfermedades respiratorias, entre ellas las EPID lo que debería facilitar el su uso en seguimientos e intervenciones como la RP (Ferrer, et al., 1996). Para el cálculo de la puntuación del SGRQ se tuvo en cuenta las instrucciones del manual de descripción y uso del SGRQ de la Universidad de Londres disponible en línea.

Luego de responder los cuestionarios se evaluó la capacidad funcional utilizando el TC6M bajo las guías de manejo de la American Thoracic Society (ATS, 2002), la prueba fue monitorizada a través de un pulsoximetro (NONIN GO2 Finger Pulse Oximeter ${ }^{\circledR}$ PN\# 9570) en el que se incluyeron datos de la distancia recorrida, Disnea de Borg modificada, frecuencia cardiaca, saturación parcial de oxígeno $\left(\mathrm{SpO}_{2}\right)$ al inicio y final de la prueba, porcentaje de desaturación durante el TC6M, Consumo de Oxigeno (Vo2) basado en la fórmula del Colegio Americano de Medicina del Deporte (CAMD) (Holland et al, 2012). En el TC6M se utilizó un pasillo con una distancia de 30 metros, en el que se incentivó a caminar lo más rápido y se le indicó el tiempo trascurrido durante la prueba minuto a minuto, se hizo un registro del número de giros y metros recorridos en los 6 minutos.

\section{Procedimiento}

Se realizó una revisión y recolección de información en las historias clínicas de los pacientes que ingresaron a RP con el fin de confirmar el diagnóstico de EPID por parte de neumología. Una vez confirmado el diagnóstico de los participantes, estos fueron citados antes de iniciar la RP para responder los cuestionarios de información sociodemográfica y clínica. De forma autoadministrada los participantes respondieron la escala MRC, el cuestionario HADS y SGRQ, luego de lo cual fueron evaluados en su capacidad funcional

La evaluación al ingreso del programa de RP fue realizada por un fisioterapeuta especialista en rehabilitación cardiaca y pulmonar y se realizó en las horas de la mañana una vez fueron registrados los datos de la historia clínica.

La recolección de las variables se registró en una base de datos diseñada en Excel 2010 y se sistematizó en el paquete estadístico SPSS Versión 24. Para el análisis de la información se presentan las variables cualitativas en frecuencias y porcentajes; mediante la prueba de Shapiro-Wilk se estableció la normalidad de las variables cuantitativas, presentándolas en media \pm desviación estándar (DE), aquellas que no cumplieron los supuestos de normalidad se presentan en mediana y amplitud intercuartil; se calculó la Oportunidad de Riesgo (OR) para la variable haber fumado y exposición al humo de leña en hombres vs mujeres y para la variable urgencias que ocasionaban hospitalización, se realizaron ajustes mediante método de Cornfield, se tuvo en cuenta un intervalo de confianza al 95\% y una significancia estadística menor de 5\%. El análisis de diferencias entre medias entre el sexo se realizaron mediante la prueba t para muestras independientes y varianzas iguales, siendo un valor $-\mathrm{p}=<0.05$ estadísticamente significativo.

En este estudio se adoptaron los principios éticos de la declaración de Helsinki, se tuvo cuenta la Resolución oo8430 de 1993 que establece las normas científicas, técnicas y administrativas para la investigación en salud en Colombia, y fue aprobado por el Comité de Ética Humana de la Institución Universitaria Escuela Nacional del Deporte, según acta IV. 4,1-01-03. Se garantizó estricta confidencialidad, autonomía y todas las medidas de seguridad necesarias para la aplicación de los instrumentos y pruebas de evaluación a los participantes del estudio. 


\section{Resultados}

De acuerdo con los resultados sociodemográficos y clínicos que se muestran en la tabla 1 , se estudiaron 23 mujeres y 18 hombres. El 83\% residentes en la ciudad principal de Cali y 17\% en municipios cercanos. En cuanto a las variables clínicas $39 \%$ manifestaron haber fumado (Media $=18.97 \pm 17.2$ paquetes/año) de los cuales solo un paciente continuaba fumando al momento de la evaluación y el $66.7 \%$ de los hombres había fumado comparado con 17.4\% de las mujeres, presentando un OR de 9.5 veces en que los hombres con EPID sean fumadores con un IC95\% (2.3-39.2) y un valor-p=0,0013. Ocho pacientes (7 mujeres) manifestaron haber estado expuestos al humo de leña, con una mediana de 13.5 años (Amplitud intercuartil 10), presentando un mínimo de 3 y un máximo de 20 años de exposición. Las mujeres presentaban un OR 7.4 veces de exposición al humo de leña que los hombres con igual exposición IC95\% (o.8 - 67.4) y un valor-p=0,06 no significativo.

La media para el IMC fue de 25.2 \pm 5.3 . Las visitas a urgencias por complicaciones respiratorias en el último año se presentaron en 27 pacientes con una media de 1,85 \$3,2; en el mismo periodo, 19 pacientes fueron hospitalizados por complicaciones respiratorias, encontrando que de los pacientes que visitaron urgencias fueron hospitalizados quienes tenían un OR de 26 con respecto a pacientes que no visitaban urgencias con un IC95\% (3.6 - 174.5); adicionalmente, se encontró que un $66.7 \%$ de los pacientes que visitaban urgencias posteriormente se hospitalizaban (Media $=9.7 \pm 14,4$ días hospitalizados y un rango mínimo de 3 y máximo de 60 días). Los datos encontrados en la espirometría en porcentaje del predicho fueron VEF1 $66 \pm 12.4$, CVF $62 \pm 12.1$ y VEF1/CVF 105.7 \pm 16.4 ; la escala de disnea MRC presento una media de $3 \pm 1.4$.

De acuerdo con la tabla 2 que recoge los diagnósticos al momento del ingreso, la EPID de origen inespecífico y la FPI fueron los más frecuentes. Así, los fármacos usados correspondían a inhaladores $\mathrm{B}_{2}$ de acción corta y anticolinérgicos inhalado de acción corta con un $48.6 \%$ de los casos, glucocorticoides por vía oral $29.7 \%$, mucolíticos y anti-inflamatorios inhalados con $24.3 \% .20$ pacientes usaban oxigeno domiciliario más de 15 horas/día, la prevalencia de uso de oxígeno domiciliario para hombres fue de $44.4 \%$ y $52.2 \%$ en mujeres, sin embargo, no hubo diferencias significativas respecto a su uso según el sexo.

En cuanto a las variables de la capacidad funcional obtenidos en el TC6M que se describen en la tabla 3, la distancia recorrida para los pacientes fue de 348.2 metros \pm 109 y una desaturación promedio de $7.4 \pm 6.7$.

Tabla 2.

Diagnóstico al ingreso

\begin{tabular}{lcc}
\multicolumn{1}{c}{ Tipo } & Frecuencia & $\%$ \\
\hline EPID inespecífico & 21 & 51.2 \\
\hline Fibrosis Pulmonar Intersticial & 11 & 26.8 \\
\hline Neumonitis Intersticial & 2 & 4.9 \\
\hline Lupus & 2 & 4.9 \\
\hline Neumoconiosis & 2 & 4.9 \\
\hline
\end{tabular}

Tabla 1.

Características sociodemográficas y clínicas

\begin{tabular}{lll}
\hline Características & $\mathrm{n}=41$ & $\%$ \\
\hline Estrato socioeconómico & 7 & 17,1 \\
1 & 11 & 26,8 \\
2 & 15 & 36,6 \\
3 & 6 & 14,6 \\
4 & 2 & 4,9 \\
5 & & \\
\hline Estado civil & 20 & 48,8 \\
Casado & 6 & 14,6 \\
Soltero & 3 & 7,3 \\
Separado & 7 & 17,1 \\
Unión libre & 5 & 12,2 \\
Viudo & & \\
Antecedente de fumador & 16 & 39 \\
Si & 25 & 61 \\
No & & \\
Visitas a urgencias en el último año & 27 & 66 \\
Si & 14 & 34 \\
No & & \\
\hline Indice de masa muscular (IMC) & 3 & 7,3 \\
Delgadez & 19 & 46,3 \\
Normal & 13 & 31,7 \\
Sobrepeso & 6 & 14,6 \\
Obesidad & & \\
\hline & & \\
& &
\end{tabular}




\section{Tabla 3.}

Resultados en el Test de caminata de los 6 minutos

\begin{tabular}{lc}
\multicolumn{1}{c}{ Variable } & Media \pm DE \\
\hline Distancia Recorrida & $348.2 \pm 109$ \\
\hline SpO2 inicio & $94.8 \pm 3.1$ \\
\hline SpO2 Final & $87.4 \pm 7.9$ \\
\hline Borg Inicio & $0.4 \pm 0.7$ \\
\hline Borg Final & $2.1 \pm 2$ \\
\hline \% Desaturación & $7.4 \pm 6.7$ \\
\hline Vo2e & $8.9 \pm 1.9$ \\
\hline METS & $2.5 \pm 0.6$ \\
\hline
\end{tabular}

Nota: Valores expresados en media \pm (DE=desviación estándar). $\mathrm{SpO}_{2}$ : Saturación parcial de oxígeno en TC6M; VOze: Consumo de oxígeno pico estimado en TC6M; METS: Equivalente metabólico en TC6M.

Los resultados de la encuesta HADS mostraron en ansiedad una media de 6.5 \pm 4.3 y una depresión de 5.1 \pm 3.7. La calidad de vida relacionada con la salud evaluada con el cuestionario SGRQ arrojó Medias así: síntomas $=48.3 \pm 21.5$, actividades con mayor compromiso $=57 \pm 21.1$ impacto $=39.2 \pm 21$ y total $=48.1 \pm 18.9$ como se muestra en la figura 1.

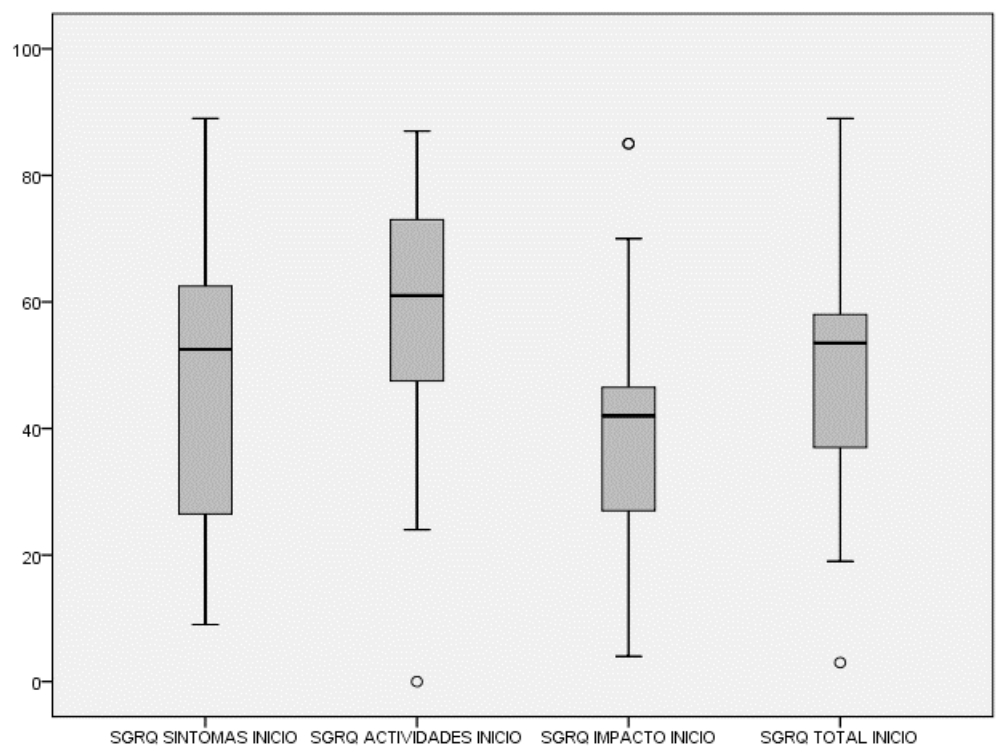

Figura 1. Calidad de vida relacionada con la salud en pacientes con EPID.

Se exploraron las diferencias en el TC6M y CVRS entre el sexo de los pacientes se muestra en la tabla 4. Se destaca una diferencia estadísticamente significativa a favor de los hombres en la distancia recorrida representada en la figura 2. 


\section{Tabla 4.}

Diferencias entre sexo en el Test de Caminata de los 6 minutos y Calidad de Vida

\begin{tabular}{|c|c|c|c|c|c|}
\hline Variables & $\begin{array}{l}\text { Ho m b res } \\
n=18\end{array}$ & $\begin{array}{l}\text { Mujeres } \\
n=23\end{array}$ & $\begin{array}{l}\text { Diferencia } \\
\text { Media } \pm \text { EE* }^{*}\end{array}$ & Valor-p & IC95\% \\
\hline Distancia Recorrida & $409 \cdot 3 \pm 95.2$ & $300.5 \pm 97.7$ & $108.8 \pm 30.4$ & 0.001 & $(47.3 / 170.3)$ \\
\hline \multicolumn{6}{|l|}{$\mathrm{SpO}_{2}$ inicio } \\
\hline $\mathrm{SpO}_{2}$ Final & $95.2 \pm 2.5$ & $94.6 \pm 3.5$ & $0.6 \pm 0.9$ & 0.5 & $(-1.4 / 2.6)$ \\
\hline \%Desaturación & $87.1 \pm 8.0$ & $87 \cdot 7 \pm 7 \cdot 9$ & $-0.6 \pm 2.5$ & 0.8 & $(-5.7 / 4.5)$ \\
\hline $\mathrm{VO}_{2} \mathrm{e}$ & $8.6 \pm 7.0$ & $6.9 \pm 6.4$ & $1.2 \pm 2.1$ & 0.6 & $(-3.1 / 5.5)$ \\
\hline \multirow[t]{2}{*}{ METS } & $9.5 \pm 2.3$ & $8.5 \pm 1.6$ & $1.0 \pm 0.6$ & 0.1 & $(-0.2 / 2.3)$ \\
\hline & $2.7 \pm 0.6$ & $2.4 \pm 0.5$ & $0.4 \pm 0.8$ & 0.09 & $(-0.05 / 0.7)$ \\
\hline \multicolumn{6}{|l|}{ SGRQ } \\
\hline Síntomas & $47 \pm 23.7$ & $49.6 \pm 19.8$ & $-2.6 \pm 7.7$ & 0.7 & $(-18.3 / 13.2)$ \\
\hline Actividades & $55.9 \pm 23.9$ & \multirow{3}{*}{$\begin{array}{l}58.1 \pm 18.6 \\
38.9 \pm 11.3 \\
48.8 \pm 11.6\end{array}$} & $-2.2 \pm 7.6$ & 0.8 & $(-17.7 / 13.2)$ \\
\hline Impacto & $39.5 \pm 27.9$ & & $0.6 \pm 7.6$ & 0.9 & $(-14.7 / 16.1)$ \\
\hline Total & $47.5 \pm 24.7$ & & $-1.3 \pm 6.8$ & 0.9 & $(-15.2 / 12.7)$ \\
\hline
\end{tabular}

Nota: Valores expresados en media \pm (desviación estándar). Valor $-\mathrm{p}=$ (prueba $t$ de muestras

independientes para igualdad de varianzas) ${ }^{*}$ Diferencia Media \pm EE: Diferencia de medias-Error estándar. $\mathrm{SpO}_{2}$ : Saturación parcial de oxígeno en $\mathrm{TC} 6 \mathrm{M} ; \mathrm{VO}_{2}$ e: Consumo de oxígeno pico estimado en TC6M; METS: Equivalente metabólico en TC6M.

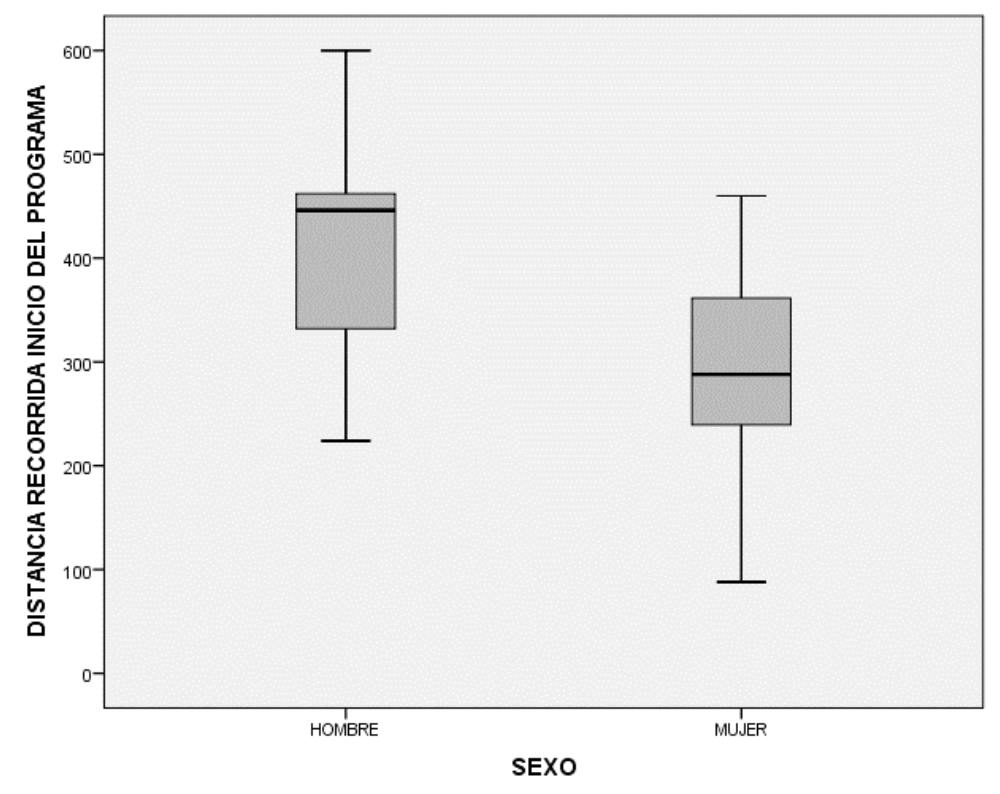

Figura 2. Diferencias en la distancia recorrida según el sexo. 


\section{Discusión}

En este estudio se describieron 41 pacientes con EPID al ingresar a un programa de rehabilitación pulmonar en la ciudad de Cali; teniendo en cuenta la remisión médica, los pacientes acudieron presentando deterioro progresivo de la capacidad funcional y disnea en las actividades diarias, la evaluación de las condiciones de ingreso permitió identificar las principales limitaciones de los pacientes (Holland et al, 2012).

La relación respecto al sexo y el tipo de EPID varía en cada estudio dependiendo la región y la clasificación de esta patología, aunque la diferencia de sexo no tiene diferencia significativa al momento de ingresar a rehabilitación pulmonar, resulta interesante describir el comportamiento del ingreso de las EPID en cuanto al sexo. En este estudio la mayoría de los pacientes fueron mujeres y se encuentran resultados similares con otros estudios (Karakatsani, et al, 2009), sin embargo, en Colombia se ha encontrado mayor cantidad de hombres vinculados a estudios de investigación (González-García et al, 2014).

La mayoría de pacientes en el estudio residían en la ciudad donde se encontraba la institución prestadora de servicios de salud al momento de ingresar al programa de RP; esto es explicado por Duran y Cols en el 2010 quienes manifestaron que el $71,42 \%$ de los programas de RP estaban ubicados en las zonas urbanas y con mayor densidad demográfica en Colombia, por lo que varios pacientes deben desplazarse desde otros municipios apartados hasta las instituciones que prestan el servicio, lo cual limita la asistencia a los programas de RP debido a la distancia y economía de estos, como consecuencia la cobertura y accesibilidad es mayor para aquellos pacientes que residen en la ciudad donde se presta el servicio de salud.

Un alto porcentaje de los pacientes masculinos tenían antecedente de haber fumado, se ha evidenciado que el consumo de tabaco aumenta el riesgo de hemorragia pulmonar, lo cual genera un aumento del daño de la membrana basal alveolar y otras alteraciones en el intersticio pulmonar; en algunas EPID es frecuente presentar este riesgo como en Histiocitosis Pulmonar, Neumonía Intersticial Descamativa, FPI y Bronquiolitis respiratoria (Ryu, Colby, Hartman, \& Vassallo, 2001), además diversos estudios relacionan el género masculino y el hábito de fumar con FPI (Baumgartner, et al., 1997; Ekström et al., 2014; Kärkkäinen et al., 2017); en este estudio el 67\% pertenecían al sexo masculino, con un OR mayor y significativo de que los hombres con EPID sean fumadores 9.5 veces más comparados con las mujeres.

Gran parte de los pacientes estudiados ingresaron bajo un diagnóstico inespecífico de EPID seguido de FPI; a nivel nacional Dueñas y Cols (1991) encontraron en su estudio diagnósticos como: Alveolitis Alérgica Extrínseca y Silicosis, asociándolo a los antecedentes laborales de los pacientes; en este caso, en nuestro estudio un reducido porcentaje presentaban EPID de origen ocupacional. A nivel internacional, el estudio de Karakatsani et al. (2009) en Grecia la Sarcoidosis, Neumonía Intersticial Idiopática y FPI fueron las más frecuentes, al igual que Jara-Palomares et al. (2009) en España, quienes encontraron Sarcoidosis, FPI y EPID asociadas a Enfermedades del Colágeno (EC); estos resultados se deben principalmente que los estudios anteriormente mencionados no fueron realizados en programas de RP, lo que supone una diferencia importante en la remisión de pacientes a estos programas.

En Colombia y gran parte de América Latina la disponibilidad de pruebas diagnósticas complementarias tales como la tomografía de tórax, Difusión de Monóxido de Carbono (DLCO) y biopsias son limitados debido a su alto costo y accesibilidad (Curbelo, 2013), 
sumado a esto la consideración innecesaria de realizar otras pruebas diagnósticas, incluido el TC6M y ecocardiograma por algunos especialistas, lo cual limita el diagnóstico y tratamiento de estos pacientes ( Varela et al., 2015), siendo también una limitante de este estudio ya que solo se pudo contar con la tomografía de alta resolución y radiología para el diagnóstico de la enfermedad, sin embargo un número importante de pacientes presentaban EPID inespecífico ya que no tenían otras pruebas complementarias como la biopsia, DLCO o la pletismografía.

Dos terceras partes de los pacientes habían visitado urgencias por complicaciones respiratorias, y casi la mitad tuvieron hospitalizaciones por esta misma complicación, lo que puede relacionarse con el deterioro progresivo de su enfermedad, que implica exacerbaciones agudas, posibles infecciones respiratorias, embolia pulmonar y/o insuficiencia cardiaca (Kim et al., 2006); estas exacerbaciones agudas son consideradas la mayor causa de mortalidad en estos pacientes (Raghu et al, 2011), en su estudio con 147 pacientes, Kim et al. (2006) evidenciaron la frecuencia de exacerbaciones agudas en pacientes con FPI de $8,5 \%$ en un año y de 9,6\% a los 2 años; estos valores se encuentran por debajo de los encontrados en el presente estudio debido posiblemente a que los pacientes vinculados en este estudio presentaban mayor deterioro clínico y funcional y presentaban mayor frecuencia de comorbilidades.

En cuanto a la fármacos los Beta 2 de corta - larga acción y anticolinérgicos, pueden mejorar la sintomatología, los glucocorticoides son los fármacos más empleados en la FPI para mejorar la síntesis de colágeno, sin embargo, sigue sin demostrarse aún eficacia terapéutica (Fernández et al, 2009); en otros estudios se observa el uso de esteroides, antifibróticos o antiinflamatorios los cuales buscan un mejor tratamiento a las EPID, no obstante, en el estudio la mayoría de los fármacos estaban destinados a aliviar la sintomatología de los pacientes. Respecto al uso de oxígeno domiciliario el $49 \%$ lo requiere y se conoce que el aporte de este debe proporcionarse si la Presión Arterial de Oxigeno Paoz en reposo es menor de 55mmHg, sin embargo, en este estudio una limitante fue que solo se tuvo en cuenta la SpO2 por pulsoximetría (Fernández et al, 2009); es importante tener en cuenta que la oxigenoterapia domiciliaria ha mostrado que puede limitar aún más la capacidad funcional y calidad de vida en pacientes con EPOC comparado con pacientes que no la usan (Kim et al, 2006; Betancourt-Peña, \& Tonguino-Rosero, 2016), estas implicaciones deben ser estudiadas más a profundidad en futuras investigaciones dado que podrían explicar el deterioro pronunciado de la funcionalidad en los pacientes con EPID.

La disnea al esfuerzo es uno de los síntomas junto a la tos seca que más afecta a las personas con EPID, apreciándose el progreso de esta al realizar actividades de la vida diaria, en este caso, la disnea MRC evidenció valores altos relacionados con una marcada limitación funcional, lo que no solo perjudica la capacidad aeróbica, sino también la calidad de vida de los pacientes (Betancourt-Peña, \& Hurtado Gutiérrez, 2015; Jastrzebski, et al., 2006).

Diversos autores afirman que diferentes factores se relacionan con el pronóstico de vida de los pacientes, siendo el TC6M la prueba funcional que arroja mayor información sobre esto; la $\mathrm{SpO}_{2}$ es un factor independiente de la distancia recorrida y si se presenta una caída del umbral de $\mathrm{SpO}_{2} \leq 88 \%$ aumenta la mortalidad según Casanova et al. (2011), al igual, como lo menciona Lama et al. (2003) en pacientes con FPI, si la distancia recorrida es menor de 207 metros se presenta un riesgo de mortalidad 4 veces mayor que los que caminan distancias mayores (Casanova-Macario, Velasco-Gonzáles, De Torres-Tajes, 2011), en este estudio los resultados en la Spoz se mantuvieron con un promedio de 87.4\% al final del TC6M y solo un paciente recorrió menos de 207 metros, lo que evidencia el gran compromiso funcional que presentan los pacientes con EPID al ingreso de un programa de RP; al analizar la distancia recorrida en el TC6M se observa una diferencia en la distancia de hombres y mujeres de casi 108.8 metros, lo cual se puede asociar a factores anatómicos y fisiológicos como se ha observado en TC6M realizados en pacientes sanos en países de Latinoamérica (Padrón, Flores, Pérez, \& Hernández, 200o). Se considera que es necesario profundizar en próximos estudios debido a que hay poca información detallada para brindar una explicación certera. 
Respecto a la calidad de vida relacionada con la salud, el cuestionario de SGRQ mostró ser una herramienta útil para valorar la calidad de vida de los pacientes encontrando mayor compromiso en el dominio actividades, lo cual se puede asociar a la limitación funcional e incremento de la disnea que se destaca de estos pacientes al realizar sus actividades de la vida diaria, (Jastrzebski et al 2006); los resultados en hombres y mujeres fueron muy similares, sin embargo, las mujeres tuvieron una puntuación mayor en algunos dominios; en un estudio similar pero realizado en pacientes con EPOC en el 2011 (Coleta et al, 2011), se observan resultados ligeramente mayores comprometidos en mujeres usando el cuestionario SGRQ, estos autores manifestaron que estos resultados estaban relacionados con los beneficios de la oxigenoterapia a largo plazo que puede beneficiar más a las mujeres, sin embargo, los resultados de este estudio frente a la limitación funcional y la ligera diferencia de la calidad de vida en relación a las mujeres son controversiales, debe investigarse más a fondo sobre la diferencia de la sintomatología y funcionalidad entre sexo, adherencia a la oxigenoterapia y efectos de la rehabilitación pulmonar.

Sobre la ansiedad y depresión la mayoría de los pacientes estuvieron sin sospechas a pesar de la progresión individual de su patología base; resultados similares se obtuvieron en el estudio de Naji, Connor, Donnelly y McDonnell (2006) el cual emplea el cuestionario HADS en pacientes con patologías incluyendo las EPID, mostrando una puntuación de ansiedad de 7,5 y depresión de 5,5 sin riesgo de presentar depresión, Holland et al. (2014) en su estudio, manifestaron que la ansiedad y la depresión no se relacionaban con la edad, el sexo ni el tipo de EPID, lo cual se asemeja a los resultados de este estudio, sin embargo este aspecto resulta ser necesario a evaluar siempre en pacientes con EPID.

En conclusión, la clasificación y diagnóstico de EPID en esta población se encuentra limitada estrictamente a los hallazgos obtenidos en la radiografía de tórax y la tomografía axial computarizada de alta resolución; el hábito de fumar fue un factor de riesgo prevalente en hombres que presentaban EPID, a su vez pacientes quienes habían asistido a urgencias por complicaciones respiratorias en el último año presentaron un OR significativo para hospitalizarse antes de iniciar el programa de rehabilitación pulmonar. En los pacientes estudiados, el test de caminata de los 6 minutos mostró la limitación de la capacidad funcional principalmente en las mujeres.

\section{Agradecimientos}

Los autores agradecen a los pacientes por su participación en el estudio, al grupo de investigación GIESS, la MSc. Olga Suárez y al semillero de investigación SEINCAR de la Institución Universitaria Escuela Nacional del Deporte.

\section{Conflicto De Intereses}

Los autores declaran no presentar ninguna relación de interés comercial o personal dentro del marco de la investigación que condujo a la producción del manuscrito.

\section{Colaboraciones}

Todos los autores han contribuido intelectualmente en la elaboración del documento.

Jhonatan Betancourt: Investigador principal del estudio, encargado de vincular la población de estudio, escribir la metodología, resultados y discusión.

Nathalie Torres: Profesional encargada de la escritura de la introducción, apoyo en el diseño de las tablas - descripción de resultados y escritura en la discusión.

Hugo Hurtado: Profesional encargado de apoyar el diseño y la escritura de la metodología, plan de análisis de resultados - descripción de resultados y escritura de la metodología. 


\section{Referencias}

American Thoracic Society. (2002). Guidelines for the six-minute walk test. American Journal of Respiratory and Critical Care Medicine, 166(1), 111-117. doi: 10.1164/rccm.201007-1179OC

Baumgartner, K. B., Samet, J. M., Stidley, C. A., Colby, T. V., \& Waldron, J. A. (1997). Cigarette smoking: a risk factor for idiopathic pulmonary fibrosis. American journal of respiratory and critical care medicine, 155(1), 242-248. doi: 10.1164/ ajrccm.155.1.9001319

Betancourt-Peña, J., \& Hurtado-Gutiérrez, H. (2015). Efectos de un programa de rehabilitación pulmonar en pacientes con enfermedad pulmonar Intersticial difusa. Fisioterapia, 37(6), 286-292. doi: 10.1016/j.ft.2014.12.001

Betancourt-Peña, J., \& Tonguino-Rosero, S. (2016). Impacto de la oxigenoterapia domiciliaria en la capacidad funcional de pacientes con enfermedad pulmonar obstructiva crónica. Rehabilitación, 50(1), 13-18. doi: 10.1016/j.rh.2015.10.001

Betancourt-Peña, J., Muñoz-Erazo, B. E., \& Mora-Guerra, R. V. (2015). Calidad de vida en pacientes con enfermedad pulmonar obstructiva crónica al ingreso de un programa de rehabilitación. Revista Colombiana de Rehabilitación, 14(1), 46-53. doi: 10.30788/RevColReh.v14.n1.2015.18

Casanova-Macario, C., Velasco-Gonzáles, M.D., De Torres-Tajes, J.P. (2011). La prueba de la marcha de 6 minutos en las enfermedades respiratorias crónicas. Medicina Respiratoria, 4(1), 57-67. Recuperado de http://www.neumologiaysalud.com/descargas/volumen4/vol4-n1-7.pdf

Coleta, K. D., Lima, D. F., Tanni, S. E., Silveira, L. V., Godoy, I., \& Godoy, I. (2011). Asociación del sexo con el estado de salud en respuesta a la oxigenoterapia crónica domiciliaria en pacientes con enfermedad pulmonar obstructiva crónica. Archivos de Bronconeumología, 47(8), 382-388. doi: 10.1016/j.arbres.2011.03.009

Curbelo, P. (2013). Encuesta de EPID en Latinoamérica-ALAT. Respirar, 5(2), 5-8. Recuperado de https://alatorax.org/es/ publicaciones/respirar/archivo

Dueñas, C., Londoño, A., Manzano, A. C., \& Ojeda, P. (1991). Enfermedad pulmonar intersticial difusa (EPID). Acta Médica Colombiana, 16(3). Recuperado de http://www.actamedicacolombiana.com/anexo/articulos/o3-1991-02-.pdf

Duran Palomino, D., Wilches Luna, E. C., \& Martínez Santa, J. (2010). Description and analysis of pulmonary rehabilitation care programs current state in four cities of Colombia. Revista Ciencias de la Salud, 8(1), 43-55. Recuperado de http://www.scielo.org.co/scielo.php?pid=S1692-72732010000100005\&script=sci_arttext\&tlng=en

Ekström, M., Gustafson, T., Boman, K., Nilsson, K., Tornling, G., Murgia, N., \& Torén, K. (2014). Effects of smoking, gender and occupational exposure on the risk of severe pulmonary fibrosis: a population-based case-control study. British Medical Journal Open, 4(1), eo04018. doi: 10.1136/bmjopen-2013-004018 
Fernández, D. I., Sánchez, R. P., Moreno, A. F., \& Fabrellas, E. F. (2009). Aspectos relevantes en el manejo de la enfermedad pulmonar intersticial difusa. Archivos de Bronconeumología, 45, 3-8. doi: 10.1016/So300-2896(o9)72851-0

Ferrer, M., Alonso, J., Prieto, L., Plaza, V., Monsó, E., Marrades, R., \& Antó, J. M. (1996). Validity and reliability of the St George's Respiratory Questionnaire after adaptation to a different language and culture: the Spanish example. European Respiratory Journal, 9(6), 1160-1166. doi: 10.1183/09031936.96.09061160

Fletcher, C. M. (1960). Standardised questionnaire on respiratory symptoms: a statement prepared and approved by the MRC Committee on the Aetiology of Chronic Bronchitis (MRC breathlessness score). British Medical Journal, 2(2), 1665. Recuperado de https://www.ncbi.nlm.nih.gov/pmc/articles/PMC2098438/pdf/brmedj03053-0087a. pdf

González-García, M., Chamorro, J., Jaramillo, C., Casas, A., \& Maldonado, D. (2014). Survival of patients with idiopathic pulmonary fibrosis at the altitude of Bogota (2640 m). Acta Medica Colombiana, 39(1), 15-20. Recuperado de http://www.scielo.org.co/pdf/amc/v39n1/v39n1ao6.pdf

Holland, A. E., Fiore, J. F., Bell, E. C., Goh, N., Westall, G., Symons, K., ... \& Glaspole, I. (2014). Dyspnoea and comorbidity contribute to anxiety and depression in interstitial lung disease. Respirology, 19(8), 1215-1221. doi: 10.1111/ resp. 12360

Holland, A. E., Hill, C. J., Glaspole, I., Goh, N., \& McDonald, C. F. (2012). Predictors of benefit following pulmonary rehabilitation for interstitial lung disease. Respiratory Medicine, 106(3), 429-435. doi: 10.1016/j.rmed.2011.11.014

Jara-Palomares, L., Martín-Juan, J., Gómez-Izquierdo, L., Cayuela-Domínguez, A., Rodríguez-Becerra, E., \& Rodríguez-Panadero, F. (2009). Hallazgos en el lavado broncoalveolar de pacientes con enfermedad pulmonar intersticial difusa. Estudio de una cohorte prospectiva de 562 pacientes. Archivos de Bronconeumología, 45(3), 111-117. doi: 10.1016/j.arbres.2008.04.005

Jastrzebski, D., Gumola, A., Gawlik, R., \& Kozielski, J. (2006). Dyspnea and quality of life in patients with pulmonary fibrosis after six weeks of respiratory rehabilitation. Journal of Physiology and Pharmacology, 57, 139. Recuperado de https://www.researchgate.net/profile/Jerzy_Kozielski/publication/6724401_Dyspnea_and_quality_of_life_ in_patients_with_pulmonary_fibrosis_after_six_weeks_of_respiratory_rehabilitation/links/of 3175307 c70fd3fd6000000.pdf

Karakatsani, A., Papakosta, D., Rapti, A., Antoniou, K. M., Dimadi, M., Markopoulou, A., ... \& Bouros, D. (2009). Epidemiology of interstitial lung diseases in Greece. Respiratory Medicine, 103(8), 1122-1129. doi: 10.1016/j. rmed.2009.03.001

Kärkkäinen, M., Kettunen, H. P., Nurmi, H., Selander, T., Purokivi, M., \& Kaarteenaho, R. (2017). Effect of smoking and comorbidities on survival in idiopathic pulmonary fibrosis. Respiratory Research, 18(1), 160. doi: 10.1186/s12931$017-0642-6$

Kim, D. S., Park, J. H., Park, B. K., Lee, J. S., Nicholson, A. G., \& Colby, T. (2006). Acute exacerbation of idiopathic pulmonary fibrosis: frequency and clinical features. European Respiratory Journal, 27(1), 143-150. doi: 10.1183/09031936.06.00114004 
King Jr, T. E. (2005). Clinical advances in the diagnosis and therapy of the interstitial lung diseases. American Journal of Respiratory and Critical Care Medicine, 172(3), 268-279. doi: 10.1164/rccm.200503-483OE

Lama, V. N., Flaherty, K. R., Toews, G. B., Colby, T. V., Travis, W. D., Long, Q., \& Martinez, F. J. (2003). Prognostic value of desaturation during a 6-minute walk test in idiopathic interstitial pneumonia. American Journal of Respiratory and Critical Care Medicine, 168(9), 1084-1090. doi: 10.1164/rccm.200302-219OC

López-Campos, J.L, Rodríguez-Becerra, E. (2004). Incidence of interstitial lung diseases in the south of Spain 1998-2000: The RENIA study. European Journal of Epidemiology, 19(2), 155-161. Recuperado de https://link.springer.com/ article/10.1023/B:EJEP.ooooo17660.18541.83

Naji, N.A., Connor, M.C., Donnelly, S.C., \& McDonnell, T.J. (2006). Effectiveness of pulmonary rehabilitation in restrictive lung disease. Journal of Cardiopulmonary Rehabilitation and Prevention, 26(4), 237-43. Recuperado de https:// journals.lww.com/jcrjournal/Abstract/2006/07000/Effectiveness_of_Pulmonary_Rehabilitation_in.7.aspx

Padrón, E. P., Flores, M. E., Pérez, A. P., \& Hernández, J. G. (200o). Estandarización de la prueba de caminata de 6 minutos en sujetos mexicanos sanos. Revista del Instituto Nacional de Enfermedades Respiratorias México, 13(4), 205-10. Recuperado de http://www.medigraphic.com/pdfs/iner/in-200o/inoo4d.pdf

Raghu, G., Collard, H. R., Egan, J. J., Martinez, F. J., Behr, J., Brown, K. K. \& Lynch, D. A. (2011). An official ATS/ERS/ JRS/ALAT statement: idiopathic pulmonary fibrosis: evidence-based guidelines for diagnosis and management. American Journal of Respiratory and Critical Care Medicine, 183(6), 788-824. doi: 10.1164/rccm.2009-040GL

Ryu, J. H., Colby, T. V., Hartman, T. E., \& Vassallo, R. (2001). Smoking-related interstitial lung diseases: a concise review. European Respiratory Journal, 17(1), 122-132. Recuperado de http://erj.ersjournals.com/content/17/1/122.short

Selman, M., \& Undurraga, A. (2015). Recomendaciones para el diagnóstico y tratamiento de la fibrosis pulmonar idiopática. Respirar, 1, 1-24. Recuperado de https://alatorax.org/es/publicaciones/respirar/numero/15/download/15_ file_es_K8ky4A_fpi2015-11junio2015-electronico-respirar15esp.pdf

Tejero, A., Guimerá, E. M., Farré, J. M., \& Peri, J. M. (1986). Uso clínico del HAD (Hospital Anxiety and Depression Scale) en población psiquiátrica: un estudio de su sensibilidad, fiabilidad y validez. Revista del Departamento de Psiquiatría de la Facultad de Medicina de Barcelona, 13(5), 233-238.

Torres-Duque, C., Dueñas, E., \& Caballero, A. N. (2007). Fundamentos de medicina, Neumología. Medellín-Colombia: Editorial Corporación para Investigaciones Biológicas.

Travis, W. D., Costabel, U., Hansell, D. M., King Jr, T. E., Lynch, D. A., Nicholson, A. G. \& Behr, J. (2013). An official American Thoracic Society/European Respiratory Society statement: update of the international multidisciplinary classification of the idiopathic interstitial pneumonias. American Journal of Respiratory and Critical Care Medicine, 188(6), 733-748. doi: 10.1164/rccm.201308-1483ST 
Varela, B., Tabaj, G., Enghelmayer, J. I., Quadrelli, S., Papucci, T., Pino, A., \& Fernández Casares, M. (2015). Manejo de las enfermedades intersticiales en Argentina: Una encuesta a neumonólogos. Revista Americana de Medicina Respiratoria, 15(3), 171-189. Recuperado de http://www.scielo.org.ar/pdf/ramer/v15n3/v15n3ao4.pdf

Notas

Artículo derivado de la investigación titulada Características de ingreso de pacientes con enfermedad pulmonar intersticial difusa en un programa de rehabilitación pulmonar.

\section{Información de autores:}

\section{Jhonatan Betancourt-Peña}

Fisioterapeuta. Esp. Rehabilitación Cardíaca y Pulmonar. MSc. Administración.

Facultad de Salud. Escuela de Rehabilitación Humana. Universidad del Valle.

Facultad de Salud y Rehabilitación. Institución Universitaria Escuela Nacional del Deporte.

Cardioprevent SAS.

Cali, Colombia

jhonatan.betancuourt@endeporte.edu.co

ORCID: 0000-0002-7292-7628

\section{Nathalie Torres-Del Castillo}

Fisioterapeuta.

Facultad de Salud, semillero de investigación SEINCAR. Institución Universitaria Escuela Nacional del Deporte,

ORCID: 0000-0003-2983-4722

\section{Hugo Hurtado-Gutiérrez}

Biólogo. MSc. Salud Pública. MSc. Bioestadística.

Facultad de Salud. Grupo Interdisciplinario de Estudios en Salud y Sociedad (GIESS). Institución Universitaria Escuela Nacional del Deporte.

Facultad de Salud. Universidad del Valle.

ORCID: 0000-0003-4119-1088 